\title{
RADIATION INTERACTION BETWEEN TWO SOLID HALF-SPACES*
}

\author{
BY \\ G. KLEINSTEIN \\ New York University
}

\begin{abstract}
A solution to the radiation interaction problem between two solid halfspaces is obtained. A Green's function method reduces the problem to the solution of two nonlinear integral equations for the surface temperatures which are solved by utilizing analytical and numerical techniques.
\end{abstract}

1. Introduction. The unsteady heat conduction problem in a homogeneous medium becomes a nonlinear boundary value problem once a surface condition of the form $k \partial T / \partial n=\sigma E\left(T^{4}-T e^{4}\right)$ is used. The single half-space problem, representing a solid-gas radiation interaction, has been previously considered by Jaeger [1] and by Mann and Wolf [2]. In the latter paper an integral formulation has been employed and uniqueness and existence of solutions have been extensively discussed. A similar integral approach, using a Green's function rather than the Laplace transform employed in [2], is utilized here in order to solve the problem of the surface temperature for two facing semi-infinite solids at a radiation interaction. Since the separation between the two surfaces does not enter either the differential equation or the boundary conditions it is possible to formulate a heat conduction problem for either one of the solids as if it fills up the positive halfspace, with the surface temperature of the other acting as a variable external driving force originally unknown. Under a special choice of the parameters the solid-solid radiation interaction problems may be shown to reduce to the solid-gas problem studied in [2]. The surface temperatures, which are the main concern in this paper, are obtained by applying the integral formulation at the origin of the coordinate (i.e., $x=0$ ). This results in a system of two coupled nonlinear integral equations of the Volterra type. A closer examination of the equation shows that uncoupling could be accomplished by simple algebraic manipulation of the equations.

As a numerical example the case where the material properties of the two half-spaces are the same and the initial temperatures are constants has been studied in detail. An interesting feature of this example is the fact that the order of nonlinearity is reduced. Both analytical and numerical techniques for the solution of the integral equation are presented and applied to cover the complete range of initial temperature differences between the two surfaces.

2. Analysis. The unsteady heat conduction problem for one of the interacting media, assumed to occupy the positive half-space $x>0$, is formulated as the boundary value problem consisting of the differential equation [3]

* Received October 3, 1969; revised version received January 14, 1970. This work was supported by the United States Air Force Office of Scientific Research under Grant No. AF-AFOSR-1062-67. 


$$
a_{1}^{-1} \partial T_{1} / \partial t=\partial^{2} T_{1} / \partial x^{2} \quad x>0, t>0,
$$

the initial conditions

$$
T_{1}(x, 0)=f_{1}(x) \quad x \geq 0, t=0,
$$

and the boundary conditions at the interacting surface

$$
k_{1} \partial T_{1} / \partial x=\sigma E_{1}\left[T_{1}^{4}-T_{2}^{4}(t)\right] \quad x=0, t>0 .
$$

The function $T_{1}(x, t)$ is furthermore taken to be bounded at infinity, and the standard continuity requirements on $T_{1}(x, t)$ at the origin are granted.

In the above equations $T_{1}(x, t)$ is the absolute temperature of the first medium and hence a nonnegative quantity, $a_{1}$ is the thermal diffusivity, $k_{1}$ is the thermal conductivity, $E_{1}$ the surface emissivity, and $\sigma$ is the Stefan-Boltzmann constant. In Eq. (3) $T_{1}$ and its derivative $\partial T_{1} / \partial x$ are taken as indicated at $x=0$, while $T_{2}(t)$ represents the surface temperature of the second medium-another unknown.

In formulating the boundary value problem for the second medium an advantage. may be taken of the fact that the spacing between the two media does not enter either the differential equations or the boundary and initial conditions. Thus for the second medium the boundary value problem becomes identically the same as the first, but with the subscripts 1 and 2 interchanged. In particular, there is no change in sign in Eq. (3) although physically the two surfaces are placed one in front of the other and thus their normals are pointing in opposite directions.

Reverting to a Green's function approach, the differential formulation is now transformed into an integral formulation. The Green's function which solves the auxiliary problem $\partial^{2} g / \partial x^{2}-a^{-1} \partial g / \partial t=\delta\left(x-x_{0}\right) \delta\left(t-t_{0}\right)$, vanishes at infinity, and satisfies the adiabatic boundary conditions $\partial g / \partial x=0$ at the origin is easily constructed from a fundamental solution to give

$$
\begin{aligned}
g\left(x, t / x_{0}, t_{0}\right)=(a / \pi)^{1 / 2} H\left(t-t_{0}\right)[4 & \left.\left(t-t_{0}\right)\right]^{-1 / 2} \\
\cdot & \left\{\exp \left[-\frac{\left(x-x_{0}\right)^{2}}{4 a\left(t-t_{0}\right)}\right]+\exp \left[-\frac{\left(x+x_{0}\right)^{2}}{4 a\left(t-t_{0}\right)}\right]\right\},
\end{aligned}
$$

where $H$ is the Heaviside function.

The general integral formula for the homogeneous equation in terms of the adjoint Green's function $g^{*}\left(x, t \mid x_{0} t_{0}\right)=g\left(x_{0}, t_{0} \mid x, t\right)$ has the form

$$
T\left(x_{0}, t_{0}\right)=\int_{0}^{t_{\infty}}\left(g^{*} \partial T / \partial x\right)_{x=0} d t-a^{-1} \int_{0}^{\infty}\left(T g^{*}\right)_{t-0} d x
$$

where $t_{\infty}$ is a parameter the only requirement on which is that it should be larger than $t_{0}$. Inserting the initial and boundary conditions, Eqs. (2) and (3), into Eq. (5), then interchanging the parametric $\left(x_{0}, t_{0}\right)$ with the active $(x, t)$ variables, we obtain the integral representation for $T_{i}(x, t)$

$$
\begin{aligned}
T_{i}(x, t) & =-\left(\frac{a_{i} \sigma^{2} I_{i}^{2}}{\pi k_{i}^{2}}\right)^{1 / 2} \int_{0}^{t}\left[T_{i}^{4}(0, t)-T_{J}^{4}(t)\right] \exp \left[-\frac{x^{2}}{4 a_{i}\left(t-t_{0}\right)}\right]\left(t-t_{0}\right)^{-1 / 2} d t_{0} \\
& +\left(4 a_{i} \pi t\right)^{1 / 2} \int_{0}^{\infty}\left\{\exp \left[-\frac{\left(x-x_{0}\right)^{2}}{4 a_{i} t}\right]+\exp \left[-\frac{\left(x+x_{0}\right)^{2}}{4 a_{i} t}\right]\right\} f_{i}\left(x_{0}\right) d x_{0}
\end{aligned}
$$


where $i=1$, or $2 ; J=1$, or 2 and $i \neq J$. Once $T_{i}(t)=T_{i}(0, t)$ are determined, as will be done presently, Eq. (6) becomes an explicit formula for the temperature distributions within the solid as function of $x$ and $t$.

3. Surface temperature. With the continuity conditions with respect to $x$ at the origin satisfied by $T_{i}\left(x_{i} t\right)$, the surface temperature is obtained as an integral equation by setting $x=0$ in Eq. (6). Introducing the notation

$$
\begin{gathered}
T_{i_{0}}(t)=\left(\pi a_{i} t\right)^{-1 / 2} \int_{0}^{\infty} \exp \left(-\frac{x^{2}}{4 a_{i} t}\right) f_{i}\left(x_{0}\right) d x_{0}, \\
m_{i}=\left(\frac{a_{i} \sigma^{2} E_{i}^{2}}{\pi k_{i}^{2}}\right)^{1 / 2}
\end{gathered}
$$

we may write the two integral equations for the surface temperatures $T_{1}(t)$ and $T_{2}(t)$ as

$$
T_{i}(t)+m_{i} \int_{0}^{t}\left[T_{i}^{4}\left(t_{0}\right)-T_{J}^{4}\left(t_{0}\right)\right]\left(t-t_{0}\right)^{-1 / 2} d t_{0}=T_{i_{0}}(t)
$$

where $i=1$, or $2 ; J=1$ or 2 and $i \neq J$, with $T_{i_{0}}(t)$ at most a known function of $t$.

It might appear on a first sight that Eq. (9), consisting of the two equations (i) $i=1$, $J=2$ and (ii) $i=2, J=1$, represents a coupled system of nonlinear integral equations that must be solved simultaneously. On second thought, however, uncoupling is facilitated by multiplying Eq. (9) by $m_{J}$ and then adding the two resulting equations. Written explicitly, the equivalent uncoupled two equations become

$T_{1}(t)+m_{1} \int_{0}^{t}\left\{T_{1}^{4}\left(t_{0}\right)-\left[\frac{m_{2}}{m_{1}} T_{10}\left(t_{0}\right)+T_{20}\left(t_{0}\right) \frac{m_{2}}{m_{1}} T_{1}\left(t_{0}\right)\right]^{4}\right\}\left(t-t_{0}\right)^{-1 / 2} d t_{0}=T_{10}(t)$

and

$$
\frac{m_{2}}{m_{1}} T_{1}(t)+T_{2}(t)=\frac{m_{2}}{m_{1}} T_{10}(t)+T_{20}(t)
$$

where $m_{1}$, representing the material properties parameter of the first medium, is taken as a nonzero quantity. Eqs. (10) and (11) reveal that aside from the initial temperature distributions $T_{10}$ and $T_{20}$ a single parameter

$$
\alpha=m_{2} / m_{1}
$$

defines the solutions for any material combination of the two media since the value of $m_{1}$ can always be absorbed in the time variable by using $m_{1}^{2} t$ as a new independent variable. The special case $\alpha=0$, in which Eq. (10) reduces to

$$
T_{1}\left(m_{1}^{2} t\right)+\int_{0}^{m_{1}^{2}}\left[T_{1}^{4}\left(\tau_{0}\right)-T_{20}^{4}\left(\tau_{0}\right)\right]\left(\tau-\tau_{0}\right)^{-1 / 2} d \tau_{0}=T_{10}\left(m_{1}^{2} t\right)
$$

represents the solid-gas radiation interaction case which has been thoroughly investigated by Mann and Wolf [2] using $T_{10}=0$ and $T_{20}=1$. In the following section solutions for the case $\alpha=1$ will be obtained using analytical and numerical techniques.

4. Examples. Identical matcrial properties $[\alpha=1]$. Solutions for the integral equations will be generated now for cases where the material properties of the interacting media are the same, i.e., $\alpha=1$, with different initial temperature distributions considered as a parameter. The temperature distribution will be assumed uniform; thus, $T_{10}$ and $T_{\mathbf{2 0}}$ are both constants representing the two initial temperatures of the two media respec- 
tively. It is of interest to note, before embarking on the solution of the specific examples below, that in the special case $\alpha=1$, and only in this case, the order of the dependent variable in the equation reduces from four to three. The mathematical or physical implications of this observation will not be considered here. Convenient variables for the present case are the dependent variable

$$
U_{i}=\left(T_{i}-T_{f}\right) /\left(T_{10}-T_{f}\right) \quad i=1,2
$$

and the independent variable

$$
\xi=\left(2 T_{f}\right)^{6} m^{2} t
$$

where $T_{f}=\frac{1}{2}\left(T_{10}+T_{20}\right), m=m_{1}=m_{2}$, and $U_{i}$ and $\xi$ both dimensionless quantities. With the above change of variables Eq. (10) becomes

$$
U_{1}+\int_{0}^{\xi} U_{1}\left(\xi_{0}\right)\left[1+\epsilon^{2} U_{1}^{2}\left(\xi_{0}\right)\right]\left(\xi-\xi_{0}\right)^{-1 / 2} d \xi_{0}=1
$$

and Eq. (11), $U_{1}+U_{2}=0$ where the temperature difference parameter $\epsilon$ is defined by

$$
\epsilon=\left(T_{10}-T_{20}\right) /\left(T_{10}+T_{20}\right) .
$$

A solution of Eq. (16) will be sought for a continuous $U_{1}(\xi)$. By definition (Eq. (14)) $U_{1}(\xi)$ at $\xi=0$ is equal to 1 . The behavior of $U_{1}$ in the neighborhood of $\xi=0$ and thus the nature of the continuity at $\xi=0$ can be determined from the expansion

$$
U_{1}(\xi)=1+\xi^{\alpha} \sum_{n=0}^{\infty} b_{i} \xi^{n} \quad 0 \leq \xi \leq \delta,
$$

where $\delta$ is a small parameter and $\alpha \geq 0$. Substituting the expansion (18) into the integral equation (16) and equating coefficients yields,

$$
U_{1}(\xi)=1-2\left(1+\epsilon^{2}\right) \xi^{1 / 2}+O\left(\delta^{3 / 2}\right) .
$$

Thus $U_{1}(\xi)$ belongs to the class of Hölder continuous functions with an exponent $\alpha \geq \frac{1}{2}$. We shall show now that $U_{1}$, satisfying the above continuity requirement, has the range defined by the inequality

$$
0 \leq U_{1} \leq 1 .
$$

First, at $\xi=0, U_{1}(\xi)=1$. Then for $\xi>0$ there exists a neighborhood over which $U_{1}(\xi)$ remains positive. Thus let $U_{1}(\xi)>0$ in the interval $0 \leq \xi<\xi_{1}$. Since the integral expression

$$
I(\xi)=\int_{0}^{\xi} U_{1}\left(\xi_{0}\right)\left[1+\epsilon^{2} U_{1}^{2}\left(\xi_{0}\right)\right]\left(\xi-\xi_{0}\right)^{-1 / 2} d \xi_{0}
$$

is clearly positive over this interval, then from the integral equation (16) which may be written in the form

$$
U_{1}(\xi)+I(\xi)=1
$$

it follows that $U_{1}(\xi) \leq 1$ in $0 \leq \xi<\xi_{1}$. Suppose that at $\xi=\xi_{1}, U_{1}$ vanishes. The value of the integral $I(\xi)$ at $\xi=\xi_{1}$ according to Eq. (21) is necessarily 1, i.e., $I\left(\xi_{1}\right)=1$. Consider next the neighborhood of $\xi_{1}$ for $\xi \geq \xi_{1}$. Let $U_{1}(\xi)>0$ for $\xi_{1}<\xi<\xi_{1}+\delta$. By the definition 
of the integral $I(\xi)>I\left(\xi_{1}\right)$ and therefore

$$
U_{1}(\xi)+I(\xi)>1 \text { in } \xi_{1}<\xi<\xi_{1}+\delta .
$$

Similarly if $U_{1}(\xi)<0$ for $\xi_{1}<\xi<\xi_{1}+\delta$ it follows that $I(\xi)<I\left(\xi_{1}\right)$ and therefore

$$
U_{1}(\xi)+I(\xi)<1 \text { in } \xi_{1}<\xi<\xi_{1}+\delta .
$$

Both Eq. (22) and Eq. (23) are in violation of the integral equation (21) and we must conclude that once $U_{1}$ becomes equal to zero, it will remain so indefinitely. In [2] a proof for the inequality equation (20) was given utilizing much more elaborate techniques.

If follows from Eq. (11) that $T_{1}(t)+T_{2}(t)=T_{10}+T_{20}=2 T_{f}$, and since as $t \rightarrow \infty$, $\left(T_{1}(t)-T_{2}(t)\right) \rightarrow 0$, the value of $T_{f}$, in addition to being the average temperature, represents the final temperature of either one of the two media. If furthermore it is assumed, with no loss of generality, that $T_{10}>T_{20}$, the parameter $\epsilon$ will satisfy the inequality $0 \leq \epsilon \leq 1$. A solution of $\mathrm{Eq}$. (16) for $U_{1}$ is sought now for all admitted values of the parameter $\epsilon$ with the cases $\epsilon=0$ and $\epsilon=1$ to be considered separately.

(i) The case $\epsilon=1$. This case is regular and it may be realized when $T_{20}$ is taken to its lowest limit, absolute zero.

(ii) The case $\epsilon=0$. This case is singular in the sense that with this choice $T_{10}=T_{20}$ and $U_{1}$ (Eq. (14)) becomes an ill-defined quantity. A solution of Eq. (16) for $\epsilon=0$ can be generated, however, by replacing $U_{1}$ by the variable $\bar{T}=T_{i}-T_{\text {, where now no sign }}$ restriction is imposed on $\bar{T}$. With this variable Eq. (16) becomes the homogeneous singular Volterra equation,

$$
\bar{T}(\xi)+\int_{0}^{\xi} \bar{T}\left(\xi_{0}\right)\left[1+\bar{T}^{2}(\xi)\right]\left(\xi-\xi_{0}\right)^{-1 / 2} d \xi_{0}=0,
$$

for which $\bar{T}(\xi)=0$ is clearly a solution and by standard results on uniqueness [5], [6], [7] also the only one.

It should be mentioned that the limit case $\epsilon \rightarrow 0$ (but not equal to zero) is a perfectly valid solution of $E_{(1 .}$. (16), a case to be taken up in the next section in connection with the perturbation solution.

4.1 Perturbation solution. I perturbation technique is applied to the solution of Eq. (16) for all values of the parameter $\epsilon$ over the interval $0<\epsilon<1$. Since $\epsilon$ enters the integral equation

$$
Y_{1}(\xi)+\int_{0}^{\xi} U_{1}\left(\xi_{0}\right)\left[1+\epsilon^{2} C_{1}^{2}\left(\xi_{0}\right)\right]\left(\xi-\xi_{0}\right)^{-1 / 2} d \xi=1
$$

only as $\epsilon^{2}$, it is convenient to expand $U_{1}$ with respect to the square of $\epsilon$ in the form

$$
U_{1}(\xi, \tau)=V_{0}(\xi)+\tau V_{1}(\xi)+\tau^{2} V_{2}(\xi)+\cdots+\tau^{n} v_{n}(\xi)+\cdots,
$$

where $\tau=\epsilon^{2}$.

The function $V_{0}(\xi)$, representing the limit of $U_{1}(\xi, \tau)$ as $\tau \rightarrow 0$ is defined by Eq. (16) with $\epsilon$ set equal to zero and it signifies the leading term in the expansion valid for any value of $\tau$. Substituting the series (25) into Eq. (16), with $L$ defined as the operator

$$
L V(\xi)=\int_{0}^{\xi} V\left(\xi_{0}\right)\left(\xi-\xi_{0}\right)^{-1 / 2} d \xi_{0}
$$


results in the sequence $V(\xi)$

$$
\begin{aligned}
& V_{0}(\xi)+L V_{0}(\xi)=1=L\left(\pi^{-1} \xi^{-1 / 2}\right) \\
& V_{1}(\xi)+L V_{1}(\xi)=-L V_{0}^{3}(\xi) \\
& V_{n}(\xi)+L V_{n}(\xi)=-L \sum_{i=0}^{n-1} V_{i}(\xi) \sum_{J=0}^{n-1-i} V_{J}(\xi) V_{n-1-i-J}(\xi)
\end{aligned}
$$

where the right-hand side of the zeroth equation has been written with the aid of the identity

$$
\pi^{-1} \int_{0}^{\xi} \xi_{0}^{-1 / 2}\left(\xi-\xi_{0}\right)^{-1 / 2} d \xi_{0}=1 .
$$

Taking the Laplace transform of the sequence (26) and using the notation $\mathscr{L}[V(\xi)]=\bar{V}_{n}(s)$ for the transform results in the following expressions for the $\bar{V}_{n}(s)$

$$
\begin{gathered}
\bar{V}_{0}(s)+V_{0}(s) \frac{\Gamma\left(\frac{1}{2}\right)}{s^{1 / 2}}=\frac{1}{\pi} \frac{\Gamma\left(\frac{1}{2}\right)}{s^{1 / 2}} \frac{\Gamma\left(\frac{1}{2}\right)}{s^{1 / 2}} \\
\bar{V}_{1}(s)+\bar{V}_{1}(s) \frac{\Gamma\left(\frac{1}{2}\right)}{s^{1 / 2}}=-\mathcal{L}\left[V_{0}^{3}(\xi)\right] \cdot \frac{\Gamma\left(\frac{1}{2}\right)}{s^{1 / 2}} \\
\vdots \\
\vdots \\
\bar{V}_{n}(s)+\bar{V}_{n}(s) \frac{\Gamma\left(\frac{1}{2}\right)}{s^{1 / 2}}=-\mathfrak{L}\left[\sum_{i=0}^{n-1} V_{i}(\xi) \sum_{J=0}^{n-1-i} V_{J}(\xi) V_{n-1-i-J}(\xi)\right] \cdot \frac{\Gamma\left(\frac{1}{2}\right)}{s^{1 / 2}}
\end{gathered}
$$

Solving for $\bar{V}_{n}(s)$

$$
\begin{aligned}
& \bar{V}_{0}(s)=\frac{\Gamma\left(\frac{1}{2}\right)}{s^{1 / 2}+\Gamma\left(\frac{1}{2}\right)}\left(\frac{1}{\pi} \frac{\Gamma\left(\frac{1}{2}\right)}{s^{1 / 2}}\right), \\
& \bar{V}_{1}(s)=-\frac{\Gamma\left(\frac{1}{2}\right)}{s^{1 / 2}+\Gamma\left(\frac{1}{2}\right)} \mathscr{L}\left[V_{0}^{3}(\xi)\right], \\
& \bar{V}_{n}(s)=-\frac{\Gamma\left(\frac{1}{2}\right)}{s^{1 / 2}+\Gamma\left(\frac{1}{2}\right)} \mathcal{L}\left[\sum_{1=0}^{n-1} V_{i}(\xi) \sum_{J=0}^{n-1-i} V_{J}(\xi) V_{n-1-i-J}(\xi)\right],
\end{aligned}
$$

and inverting with the aid of the convolution theorem

$$
\begin{gathered}
V_{0}(\xi)=\int_{0}^{\xi} g\left(\xi \mid \xi_{0}\right) \frac{1}{\pi} \xi_{0}^{-1 / 2} d \xi_{0} \\
V_{1}(\xi)=-\int_{0}^{\xi} g\left(\xi \mid \xi_{0}\right) V_{0}^{3}\left(\xi_{0}\right) d \xi_{0} \\
\vdots \\
\vdots \\
V_{n}(\xi)=-\int_{0}^{\xi} g\left(\xi \mid \xi_{0}\right) \sum_{i=0}^{n-1} V_{1}\left(\xi_{0}\right) \sum_{J=0}^{n-1-i} V_{J}\left(\xi_{0}\right) V_{n-1-i-J}\left(\xi_{0}\right) d \xi_{0}
\end{gathered}
$$

where

$$
g\left(\xi \mid \xi_{0}\right)=\left(\xi-\xi_{0}\right)^{-1 / 2}-\pi e^{r\left(\xi-\xi_{0}\right)} \operatorname{erfc}\left[\pi\left(\xi-\xi_{0}\right)\right]^{1 / 2}
$$

completes the solution. 
The zeroth-order term, which is given by

$$
V_{0}(\xi)=\int_{0}^{\xi}\left[\left(\xi-\xi_{0}\right)^{-1 / 2}-\pi e^{\pi\left(\xi-\xi_{0}\right)} \operatorname{erfc}\left[\pi\left(\xi-\xi_{0}\right)\right]^{1 / 2}\right] \pi^{-1} \xi_{0}^{-1 / 2} d \xi_{0},
$$

reduces with the aid of the identity (27) to

$$
V_{0}(\xi)=1-\int_{0}^{\xi} e^{\pi \xi_{0}} \operatorname{erfc}\left[\left(\pi \xi_{0}\right)^{1 / 2}\right]\left(\xi-\xi_{0}\right)^{-1 / 2} d \xi_{0},
$$

and utilizing another identity

$$
\int_{0}^{\xi} e^{\pi \xi_{0}} \operatorname{erfc}\left[\left(\pi \xi_{0}\right)^{-1 / 2}\right]\left(\xi-\xi_{0}\right)^{-1 / 2} d \xi_{0}=1-e^{\pi \xi} \operatorname{erfc}\left[(\pi \xi)^{1 / 2}\right]
$$

which is easily demonstrated by a Laplace transform, results in an explicit form for $V_{0}(\xi)$,

$$
V_{0}(\xi)=e^{r \xi} \operatorname{erfc}\left[(\pi \xi)^{1 / 2}\right] .
$$

For small values of $\xi$ Eq. (30) has the expansion

$$
V_{0}(\xi)=1-2 \xi^{1 / 2}+\pi \xi+\cdots,
$$

while asymptotically it vanishes as

$$
V_{0}(\xi) \sim \frac{1}{\pi \xi^{1 / 2}}-\frac{1}{2 \pi^{2} \xi^{3 / 2}}+\cdots
$$

For all higher orders of $V_{n}(\xi)$ resort must be made to a numerical integration of (29).

4.2 Numerical solution. For the numerical solution of the integral equation

$$
U_{1}(\xi)+\int_{0}^{\xi} U_{1}\left(\xi_{0}\right)\left[1+\tau U_{1}^{2}\left(\xi_{0}\right)\right]\left(\xi-\xi_{0}\right)^{-1 / 2} d \xi_{0}=1
$$

the interval $[0, \xi]$ is split into three regions

(a) $0 \leq \xi_{0} \leq \mu$,

(b) $\mu<\xi_{0} \leq \xi-\delta$,

(c) $\xi-\delta<\xi_{0} \leq \xi$,

where both $\mu$ and $\delta$ are arbitrary small positive constants. For the regions (a) and (c), assuming Hölder continuity for $U_{1}$ with an exponent $\geq \frac{1}{2}$ over that region, one obtains

$$
I_{a}=\int_{0}^{\mu} U_{1}\left(\xi_{0}\right)\left[1+\tau U_{1}^{2}\left(\xi_{0}\right)\right]\left(\xi-\xi_{0}\right)^{-1 / 2} d \xi_{0}=2(1+\tau)\left(\xi^{1 / 2}-(\xi-\mu)^{1 / 2}\right)+O\left(\mu^{3 / 2}\right)
$$

and

$$
I_{c}=\int_{\xi-\delta}^{\xi} U_{1}\left(\xi_{0}\right)\left[1+\tau U_{1}^{2}\left(\xi_{0}\right)\right]\left(\xi-\xi_{0}\right)^{-1 / 2} d \xi_{0}=2 U_{1}(\xi)\left[1+\tau U_{1}^{2}(\xi)\right] \delta^{1 / 2}+O\left(\delta^{3 / 2}\right) .
$$

The integral equation may be written then as

$$
\begin{aligned}
U_{1}(\xi)\left[1+2 \delta^{1 / 2}\left(1+\tau U_{1}^{2}(\xi)\right]\right. & =1-2(1+\tau)\left[\xi^{1 / 2}-(\xi-\mu)^{1 / 2}\right] \\
& \quad-\int_{\mu}^{\xi-\delta} U_{1}\left(\xi_{0}\right)\left[1+\tau U_{1}^{2}\left(\xi_{0}\right)\right]\left(\xi-\xi_{0}\right)^{-1 / 2} d \xi_{0}+O\left(\mu^{3 / 2}, \delta^{3 / 2}\right) .
\end{aligned}
$$


The advantage in this form stems from the fact that on the right-hand side the values of $U_{1}$ needed to carry out the integration are for $\xi_{0} \leq \xi-\delta$. Hence this equation gives an explicit formula for $U_{1}(\xi)$ in terms of known values of $U_{1}$ up to $\xi-\delta$.

Replacing the integral expression in Eq. (31) by a summation and using the notation $U(J) \equiv U(J \Delta \xi)$ with $\delta=\Delta \xi$ yields

$$
U_{1}(J)\left[1+2(\Delta \xi)^{1 / 2}\left(1+\tau U_{1}^{2}(J)\right)\right]=F_{J}(\tau ; \mu ; \Delta \xi)+O\left(\mu^{3 / 2} ;(\Delta \xi)^{3 / 2}\right),
$$

where $F_{J}$ is given by

$$
\begin{aligned}
F_{J}(\tau ; \mu ; \Delta \xi)= & 1-\left\{2(1+\tau)\left[J^{1 / 2}-\left(J-\mu \Delta \xi^{-1}\right)^{1 / 2}\right]+\frac{1}{2}\left[G(1)(J-1)^{-1 / 2}\right.\right. \\
& \left.\left.+G(J-1)+2 \sum_{i=2}^{J-2} G(i)(J-i)^{-1 / 2}\right]\right\}(\Delta \xi)^{1 / 2} \quad J=2,3, \cdots,
\end{aligned}
$$

and

$$
G(i)=U_{1}(i)\left[1+\tau U_{1}^{2}(i)\right] .
$$

For any value of the integer $J, F_{J}$ is determined from known values of $U_{1}$ up to $U_{1}(J-1)$, where $U_{1}(1)$ has the special value

$$
U_{1}(1)=1-2(1+\tau)\left[1-\left(1-\mu \Delta \xi^{-1}\right)^{1 / 2}\right](\Delta \xi)^{1 / 2},
$$

where $\Delta \xi \geq \mu$.

With $F_{J}$ determined the solution $U_{1}(J)$ reduces to the solution of the cubic equation (32). The nature of its solution is best seen by writing (32) as

$$
U_{1}^{3}(J)+\tau^{-1}\left[1+1 /\left(2(\Delta \xi)^{1 / 2}\right)\right] U_{1}-\left(2 \Delta \xi^{1 / 2} \tau\right)^{-1} F_{J}=0
$$

where $\tau$ and $\Delta \xi$ are necessarily nonzero.

Comparing Eq. (39) with the equation $z^{3}+a_{1} z+a_{0}=0$ and using the standard criteria for cubic equations [8, p. 17] shows that there is only one real root to Eq. (32) for any value of $J$.

In Fig. (1) the dimensionless temperature $U_{1}$, as computed from Eq. (32), is shown as a function of $\xi$ using $\tau$ as a parameter. In these calculations $\mu$ was taken equal to $\Delta \xi$ and thus the solution is good to $O\left(\Delta \xi^{3 / 2}\right)$. As a convergence check three different values of $\Delta \xi$ have been used, i.e., $\Delta \xi=1.6 \times 10^{-3}, 4.0 \times 10^{-4}$ and $4.0 \times 10^{-5}$, without significantly changing the result. In the same figure the zeroth-order analytical solution (Eq. (30)) has been also included where it is appropriately seen to represent the limit of $U_{1}(\xi, \epsilon)$ as $\epsilon$ approaches zero.

In Fig. (2) results are shown for larger values of $\xi$. The spread of $\xi$ as a function of the parameter $\epsilon$ is indicated there as the hatched area under the $V_{0}(\xi)$ line. This spread, aftcr an initial swalling zone, rapidly diminishes and at values of $\xi \sim 1.0$ narrows down into a line. At about the same neighborhood the two-term asymptotic solution (Eq. (30b)) becomes applicable. The one-term asymptotic solution has been also included there for comparison.

The physical interpretation to the interaction process could be presented in terms of a relaxation time defined as the time required for the temperature difference $T_{1}-T$, to drop to a fraction $\beta$ of its original value $T_{10}-T_{1}$. With this definition the relaxation time is given implicitly as 


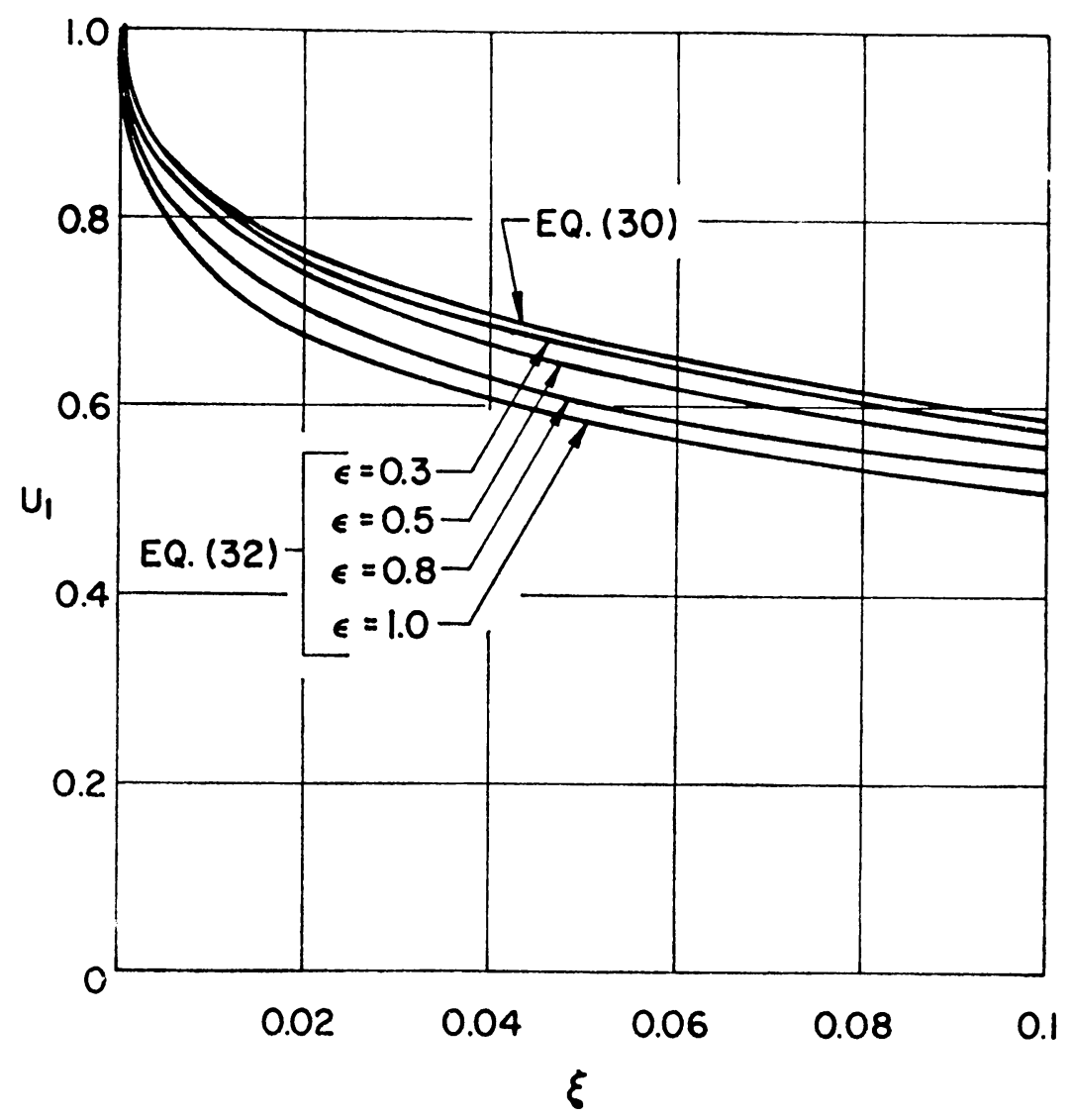

Fri. 1. The dimensionless temperature $C_{t}$ as a function of $\xi$ with $\epsilon$ as a parameter.

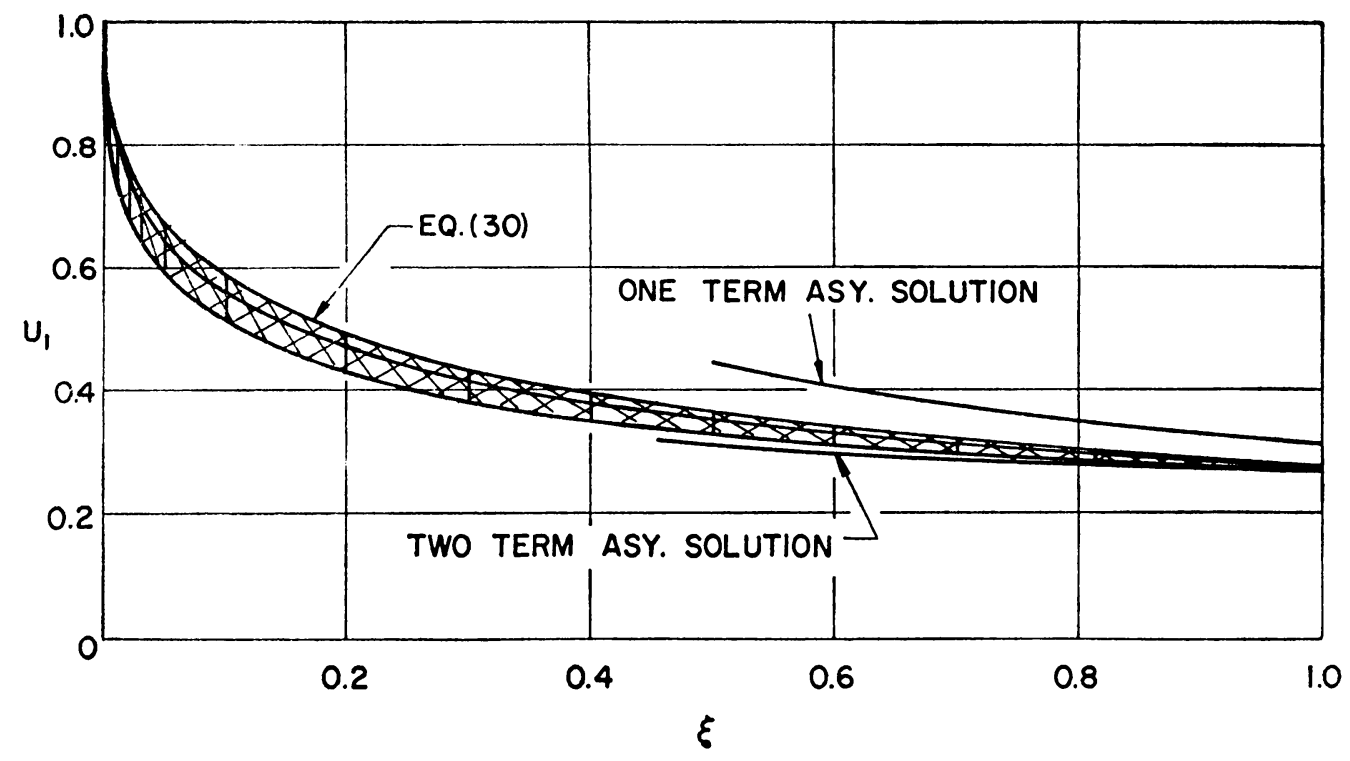

Fı. 2. The dimensionless temperature $U_{1}$ as a function of $\xi$ with $\epsilon$ as a parameter. 


$$
U_{1}\left(\xi_{\beta}\right)=\beta
$$

where for $\beta=\frac{1}{2}$ the numerical solution yields, with deviations due to $\epsilon$ effects, $\xi_{\beta}=$ $0.15+.05$. Using dimensional variables the relaxation time is given by

$$
\xi_{\beta}=\frac{\left(2 T_{f}\right)^{6} a \sigma^{2} E^{2}}{\pi k^{2}} t_{\beta},
$$

or by introducing the definition for the thermal diffusivity $a=k / \rho C_{p}$ (where $\rho$ is the density and $C_{p}$ the specific heat at constant pressure) and after rearranging as,

$$
t_{\beta}=2^{-6} \pi \xi_{\beta} a \frac{\left(\rho C_{p} T_{f}\right)^{2}}{\left(\sigma E T_{f}^{4}\right)^{2}} .
$$

In (38a) $\rho C_{p} T_{f}$ is a measure of the heat capacity per unit volume of the solid and $\sigma E T_{f}^{4}$ is a measure of the radiative heat transfer per unit surface. Excluding the coefficient of proportionality $\xi_{\beta}$ which has been determined numerically by the analysis, the relation (38a) can be also derived directly from energy considerations applied to an area element $A$ of the interacting surface. In a time $t_{\beta}$ an isotherm $\beta$ penetrates into the solid to a depth $\sim\left(a t_{\beta}\right)^{1 / 2}, a$ being the thermal diffusivity. The energy released in the solid in this time interval is $\sim \rho C_{p} T_{f} A\left(a t_{\beta}\right)^{1 / 2}$; while the energy removed at the surface by radiation is $\sim \sigma E T^{4} A t_{\beta}$. A proportionality of these two energies results in a relation of the form (38a).

5. Summary and conclusions. The radiation interaction between two media has been formulated with the aid of a Green's function and it has been shown that the surface temperatures satisfy a system of two nonlinear integral equations of the Volterra type. By a simple algebraic manipulation the system has been uncoupled and solutions for the case where the material properties of the two media are the same have been obtained with the results depicted in Figs. (1) and (2). The spread in the dependent variable $U_{1}=\left(T_{1}-T_{f}\right) /\left(T_{10}+T_{20}\right)$ is seen to be small, with the maximum value of about $15 \%$ reached between $\xi=0.05$ and $\xi=0.3$. Thus the zeroth solution may serve as a good approximation for the solution, viz.

$$
U_{1} \sim e^{\pi} \operatorname{erfc}\left[(\pi \xi)^{1 / 2}\right]
$$

for any value of $\epsilon$.

The physical aspects of the interaction are obtained in terms of a relaxation time $t_{\beta}$ (Eq. (38a)). The relaxation time was shown to increase with the heat capacity of the solid $\left(\rho C_{p} T_{f}\right)$ and with its thermal diffusivity, while it decreases with the ability of the surface to radiate $\left(\sigma E T_{f}^{4}\right)$. Another feature of the solution is the fact that $t_{\beta}$ is very strongly dependent on the temperature level of the interaction, as determined by $T_{s}=0.5\left(T_{10}+T_{20}\right)$, and only weakly on the temperature difference. The effect of the temperature difference enters through the parameter $\epsilon$, which has a small insignificant effect on the numerical value of $\xi_{\beta}$, while the interaction level $T_{f}$ reduces the relaxation time by a factor $\left(T_{f}\right)^{-6}$. This last remark could be better appreciated by considering the two interaction problems (a) $T_{10}=1000^{\circ} \mathrm{K} ; T_{20}=0^{\circ} \mathrm{K}$, and (b) $T_{10}=1500^{\circ} \mathrm{K} ; T_{20}=$ $500^{\circ} \mathrm{K}$, with both materials being the same. The maximum temperature drop in the two cases is $500^{\circ} \mathrm{K}$; however, the time required in (b) to drop $250^{\circ} \mathrm{K}$ as compared with the time required in (a) to drop $250^{\circ} \mathrm{K}$ is $2^{6}=64$ times faster. 


\section{REFERENCES}

[1] J. C. Jaeger, Conduction of heat in a solid with a power law of heat transfer at its surface, Proc. Cambridge Philos. Soc. 46, 634-641 (1950)

[2] W. R. Mann and F. Wolf, Heat transfer between solid and gasses under nonlinear boundary conditions, Quart. Appl. Math. 9, 163-184 (1950)

[3] H. S. Carslaw and J. C. Jaeger, Conduction of heat in solids, 2nd ed., Clarendon Press, Oxford, 1959

[4] J. H. Roberts and W. R. Mann, On a certain nonlinear integral equation of the Volterra type, Pacific J. Math. 1, 431-445 (1951)

[5] A. Friedman, Partial differential equations of parabolic type, Prentice-Hall, Englewood Cliffs, N. J., (1964) (especially Chapter 6)

[6] A. Friedman, On integral equations of Volterra type, J. Analyse Math. 11, 381-413 (1963)

[7] K. Padmavally, On a non-linear integral equation, J. Math. Mech. 7, 533-555 (1958)

[8] M. Abramowitz and I. A. Stegun (Editors), Handbook of mathematical functions with formulas, graphs, and mathematical tables, Nat. Bur. Standards Appl. Math. Series, 55, Superintendent of Documents, U. S. Government Printing Office, Washington, D. C., 1964; 3rd printing with corrections, 1965, p. 17 